Manuel Fernández González

Mónica Fernández Valencia

María Ángeles Manso Huerta

M. Paz Gómez Rodríguez

M. ${ }^{a}$ Carmen Jiménez Recio

Faustino del Coz Díaz

C.P.R.P.M. Mixta de Gijón.

Correspondencia:

Manuel Fernández González

CPR Mixta de Gijón

Departamento de Fisioterapia

c/ San Nicolás, 47

33210 Gijón

Tel.: 9851439 00, ext. 88031

Fax: 985143878

\section{Trastornos musculoesqueléticos en personal auxiliar de enfermería del Centro Polivalente de Recursos para Personas Mayores "Mixta" de Gijón - C.P.R.P.M. Mixta}

\author{
Musculoskeletal disorders in \\ nursing assistants from the Resource \\ Polyvalent Centre for the Elderly \\ "Mixta" gijon - C.P.R.P.M. MIXTA
}

\section{RESUMEN}

La elaboración de la Guía de Detección, Prevención e Intervención en

Patologias Musculoesqueléticas ha puesto de relieve el desconocimiento que, hasta el momento, se tenía en el Centro Polivalente de Recursos para Personas Mayores Mixta de Gijón (CPR Mixta) acerca de la incidencia de trastornos musculoesqueléticos (TME) entre los trabajadores auxiliares de enfermería que desempeñan su labor profesional en este centro. Con el fin de realizar una breve y modesta aproximación a la situación actual de los TME entre los profesionales del CPR Mixta se ha llevado

a cabo un cuestionario que, sin ser exhaustivo, pretende configurar el panorama actual y abrir la puerta a futuras investigaciones o estudios de las diversas categorías profesionales.

PALABRAS CLAVE: guía de detección, trastornos musculoesqueléticos, CPR Mixta, auxiliares de enfermería.

\section{ABSTRACT}

The making of the Guide of Detection, Prevention and Intervention in Musculoskeletal Pathologies has shown the lack of knowledge that there's in the CPR Mixta about the incidence of musculoskeletal disorders among nursing assistants who perform their professional work in this center.

In order to make a brief and modest approach to the current situation of MSDs among CPR Mixta professionals, this group has conducted a questionnaire which is intended to know the current outlook and open the door to future research or studies of the different professional categories.

KEYWORDS: Guide of detection, musculosketal disorders, CPR Mixta, nursing assistants.

\section{- INTRODUCCIÓN}

Los trastornos musculoesqueléticos (TME) de origen laboral son alteraciones que sufren estructuras corporales como los músculos, articulaciones, tendones, ligamentos, nervios, huesos y el sistema circulatorio, causadas o agravadas fundamentalmente por el trabajo y los efectos del entorno en el que éste se desarrolla. La mayor parte de los TME son trastornos acumulativos resultantes de una exposición repetida, durante un período de tiempo prolongado, a factores de riesgo biomecánico y organizacionales. Tales trastornos afectan principalmente a la espalda, cuello, hombros y extremidades superiores, aunque también pueden afectar a las inferiores. Los diagnósticos más frecuentes son las tendinitis, epicondilitis, síndrome del túnel carpiano, lumbalgias, etc.

Los factores biomecánicos y organizacionales, así como los factores psicosociales e individuales, son determinantes en la aparición de TME. La combinación de varios de ellos incrementa considerablemente el riesgo de padecer un TME.

Los TME son el problema de salud relacionado con el trabajo más común en Europa (1).

En los últimos años se ha producido un gran incremento de este tipo de trastornos, que afectan a todos los sectores profesionales con independencia de la edad y el sexo de los trabajadores.

Los TME tienen importantes consecuencias sobre el individuo. Entre otras, ver mermada su calidad de vida considerablemente debido al dolor y sufrimiento que provocan, así como la pérdida de ingresos económicos derivada de la necesidad del trabajador de acogerse (en muchos casos de forma reiterada) a una baja laboral. Sin embargo, los efectos negativos de los TME no sólo se reducen al trabajador, sino que también afectan a las empresas e instituciones en las que estas personas trabajan y, como consecuencia, a las economías de los distintos estados. Los TME son la 
principal causa de absentismo laboral en prácticamente todos los estados miembros de la Unión Europea, reducen la rentabilidad de las empresas y aumentan además los costes sociales públicos (el $40 \%$ de los costes económicos que tienen las enfermedades y los accidentes de trabajo se deben precisamente a los TME).

Casi el 24\% de los trabajadores de la Unión Europea afirma sufrir dolor de espalda, y el $22 \%$ se queja de dolores musculares.

Según la Agencia Europea para la Seguridad y Salud en el Trabajo, los TME afectan a una cuarta parte de la población europea (el 25\% de los trabajadores sufren dolores de espalda y el $23 \%$ se quejan de dolores musculares). Conforme a los datos del Eurostat, el coste económico de los TME en Europa representa el 1,6\% del PIB (205 107 millones de euros al año).

En Espańa, la VI Encuesta Nacional de Condiciones de Trabajo realizada por el Instituto Nacional de Seguridad e Higiene en el Trabajo (INSHT) revela que el 74,2\% de los trabajadores encuestados señala sentir alguna molestia que achaca a posturas y esfuerzos derivados del trabajo que realiza (3).

Entre las molestias más frecuentes figuran las localizadas en la zona baja de la espalda (40,1\%), la nucalcuello (27\%) y la zona alta de la espalda (26,6\%).

La elaboración de la Guia de Detección, Prevención e Intervención en Patologías Musculoesqueléticas ha puesto de relieve el desconocimiento que, hasta el momento, se tenía en el CPR Mixta acerca de la incidencia de TME entre los trabajadores que desempeñan su labor profesional en este centro.

Con el fin de realizar una breve y modesta aproximación a la situación actual de los TME entre los profesionales del CPR Mixta se ha llevado a cabo un cuestionario que, sin ser exhaustivo, pretende configurar el panorama actual y abrir la puerta a futuras investigaciones o estudios de las diversas categorías profesionales. Los resultados del mismo se presentan a continuación.

\section{- MÉTODOS}

\section{Población}

El cuestionario fue dirigido hacia profesionales auxiliares de enfermería trabajadores del centro a turnos y con trabajo nocturno, que desarrollan su labor profesional en las plantas de residentes con plaza calificada de asistida. La media de edad de estos profesionales es de 49,04 ańos. No se realizaron distinciones entre sexos debido a que este colectivo tiene una elevada dominancia femenina y no resultan representativos el número de profesionales auxiliares de enfermería hombres.

\section{Metodología}

El cuestionario fue distribuido a los profesionales a través de la responsable de área asistencial durante la segunda quincena de noviembre. El plazo de realización fue de 3 días, y se recogieron un total de 49 encuestas cumplimentadas por otros tantos profesionales.

\section{Objetivos}

El objetivo de la aplicación de este cuestionario de lesiones musculoesqueléticas es realizar una breve aproximación a la realidad de los TME en el CPR Mixta.

Se trata de conocer en qué medida los profesionales, en concreto, auxiliares de enfermería, están afectados por síntomas físicos, organizati- vos y psicosociales relacionados con los TME. Los resultados obtenidos ayudan a clarificar esta situación y, en consecuencia, pueden servir para planificar medidas de prevención e intervención concretas.

\section{Elaboración de la encuesta}

El objetivo fundamental era elaborar un instrumento de fácil manejo que permitiera recoger información de una forma rápida y sencilla.

De la bibliografía revisada resultaron especialmente atractivos el Cuestionario nórdico de Kuorinka (1987) y el Cuestionario de lesiones musculoesqueléticas de UGT Canarias e Instituto Canario de Seguridad Laboral (ICASEL) (Anexo I).

\section{Cuestionario NóRdico}

El Cuestionario Nórdico Estandarizado (4), también conocido como Cuestionario de Kuorinka, es un cuestionario estandarizado para la detección y análisis de síntomas musculoesqueléticos, aplicable en el contexto de estudios ergonómicos o de salud ocupacional con el fin de detectar la existencia de síntomas iniciales, que todavía no han constituido enfermedad o no han llevado aún a consultar al médico.

Su valor radica en que nos da información que permite estimar el nivel de riesgos de manera proactiva y nos permite una actuación precoz.

Las preguntas se concentran en la mayoría de los síntomas que, con frecuencia, se detectan en diferentes actividades económicas. La fiabilidad de los cuestionarios se ha demostrado aceptable. Algunas características específicas de los esfuerzos realizados en el trabajo se muestran en la frecuencia de las respuestas a los cuestionarios.

El Cuestionario Nórdico se centra fundamentalmente en los síntomas físicos de los TME. Los factores organizativos y psicosociales relacionados con los TME son de gran importancia e interés de cara a realizar una abordaje lo más integral posible, por lo que el cuestionario administrado en el CPR Mixta se complementó con el Cuestionario de Lesiones Musculoesqueléticas de UGT Canarias e Instituto Canario de Seguridad Laboral (ICASEL).

\section{Cuestionario de Lesiones Musculoesqueléticas de UGT Canarias E Instituto Canario de Seguridad Laboral (5)}

Se trata de una herramienta corta y de fácil aplicación, desarrollada por UGT-Canarias, a través de la Secretaría de Salud Laboral y Medio Ambiente. Pertenece a la guía Lesiones músculo-esqueléticas de espalda, columna vertebral y extremidades. Su incidencia en la mujer trabajadora. Técnicos Auxiliares de Enfermería, Centros de Rehabilitación y Residencias de la Tercera Edad (http://www.saludlaboralcanarias.org/).

Este cuestionario es meramente exploratorio y está orientado hacia centros sanitarios y residencias de personas mayores. Puede ser aplicado por trabajadores con mínimos conocimientos en prevención.

De este cuestionario se incluyeron los siguientes ítems: factores que se presentan en el trabajo, esquema de las partes del cuerpo, factores procedentes de las exigencias de la tarea, factores procedentes de las condiciones físicas del entorno, factores sociales y organizativos que generan más carga mental, características de la carga, esfuerzo físico necesario, características del medio de trabajo y exigencias de la actividad.

\section{Interpretación de resultados}

Una vez recogidos todos los cuestionarios se extrajo la información relevante de cada uno de los ítems obteniendo datos de tipo porcentual que se representaron gráficamente. 


\section{- RESULTADOS}

El tipo de trabajo desarrollado por los auxiliares de enfermería está considerado como uno de los de mayor predisposición a padecer algún tipo de TME, por lo que es de esperar que así ocurra en el CPR Mixta.

Los resultados obtenidos así lo confirman: más de la mitad de los encuestados, un 57,4\%, manifiesta haber padecido molestias y/o dolores en los últimos 3 meses. Las zonas más afectadas son la espalda, el cuello y el hombro izquierdo. Igualmente, esta situación se produce cuando nos referimos a la existencia de molestias y/o dolores en los últimos 7 días; en este caso, las zonas más afectadas siguen siendo cuello, espalda y hombro izquierdo, en este orden.

En un $31,1 \%$, la duración de las molestias es permanente, siendo el nivel de intensidad de éstas en torno al 3 (de una escala de 0 a 4 ). El 11\% de los encuestados manifiesta tener una intensidad máxima de molestias y/o dolor. El 26,27\% de los encuestados ha precisado tratamiento médico en los últimos meses. Dicho tratamiento médico no se extiende a la totalidad de afectados por molestias y/o dolores en los últimos 3 meses (57,4\%), lo cual puede indicar la no necesidad de éste o bien una posible automedicación. Resulta significativa la ausencia de resultados en cuanto a la duración de la incapacidad en los últimos 3 meses (bajas laborales); ello puede llevar a pensar que algunos TME, por su intensidad y duración, no precisan necesariamente una baja laboral o bien que el profesional decide no acogerse a la baja laboral por las consecuencias fundamentalmente económicas que acarrea (el 11\% de los encuestados había manifestado una intensidad de dolor máxima).

Los factores en el trabajo relacionados con los TME más valorados por los encuestados son las posturas forzadas, la manipulación de cargas, el ritmo de trabajo elevado, los movimientos repetidos y el estrés, en este orden.

Los factores procedentes de las exigencias de la tarea relacionados con los TME que los encuestados consideran como más importantes son la responsabilidad por la salud o la seguridad de otros, la jornada prolongada, turnos y trabajo nocturno y la atención sostenida sobre una o más fuentes de información, por este orden. Las condiciones físicas del entorno que a los profesionales les parecen más inadecuadas en el puesto de trabajo son los olores, las condiciones ambientales (calor, humedad, calidad del aire, etc.) y la limpieza.

Los contactos sociales (relación con usuarios y clientes), los conflictos (en el grupo, entre grupos y entre personas), las exigencias sociales (responsabilidad en relación con la salud y el bienestar públicos) y la situación económica (mercado laboral) son los factores sociales y organizativos que generan más carga mental en el puesto de trabajo.

En cuanto a las características de la carga, el estar colocada de tal modo que debe sostenerse o manipularse a distancia del tronco o con torsión o inclinación de éste, y el ser pesada, grande, voluminosa y difícil de sujetar son los aspectos relacionados con los TME más valorados por los encuestados. La gran mayoría, 71,42\%, considera que el esfuerzo físico necesario puede acarrear un movimiento brusco de la carga, mientras que para el 55,10\%, el esfuerzo físico necesario es demasiado importante. Asimismo, según la mayoría de los encuestados, la situación o el medio de trabajo no permite al trabajador la manipulación manual de cargas a una altura segura y en una postura correcta. Resultan significativos además la consideración por parte de los encuestados de la existencia en su centro de trabajo de una temperatura, humedad o circulación del aire inadecuada, y la presencia de desniveles en el suelo o plano, lo que implica la manipulación de la carga en niveles diferentes.

Los esfuerzos físicos frecuentes o prolongados en los que interviene en particular la columna vertebral son la exigencia de la actividad más puntuada por los encuestados $(83,67 \%)$, seguido de la consideración como insuficiente del período de recuperación.

\section{- REPERCUSIONES FISICAS DE LOS TRASTORNOS MUSCULOESOUELÉTICOS EN EL PERSONAL AUXILIAR DE ENFERMERÍA DEL CPR MIXTA. GRÁFICOS REPRESENTATIVOS}

El gráfico 1 indica el porcentaje de encuestados que ha presentado molestias en las distintas zonas corporales susceptibles de TME en los últimos 3 meses. La zona corporal más afectada es la espalda, seguida del cuello y hombro izquierdo.

Resulta significativo que de estos datos porcentuales se deduce que un $57,4 \%$ de la muestra encuestada informa de la presencia de molestias $y / 0$ dolores en los últimos 3 meses.

El gráfico 2 indica el porcentaje de afectados por molestias y/o dolores de los TME en las distintas zonas corporales (miembro superior y tronco y cabeza) durante intervalos de tiempo (de 1 a 7 días, de 8 a 30 días, más de 30 días o de forma permanente).

Resulta significativo los elevados porcentajes obtenidos en molestias y/o dolores que se producen de forma permanente, una media de un $31 \%$, siendo el cuello la zona corporal en la que hay un mayor número de encuestados afectados de forma permanente (47\%).

El gráfico 3 indica el porcentaje de afectados por molestias y/o dolores de los TME en las distintas zonas corporales (miembro superior y tronco y cabeza) en distintos niveles de intensidad $(0,1,2,3,4$, donde 0 es la ausencia de dolor y 4 la intensidad más elevada de dolor).

Resultan significativos los elevados porcentajes obtenidos en molestias y/o dolores en la espalda con una intensidad de 3 (28,57\%).

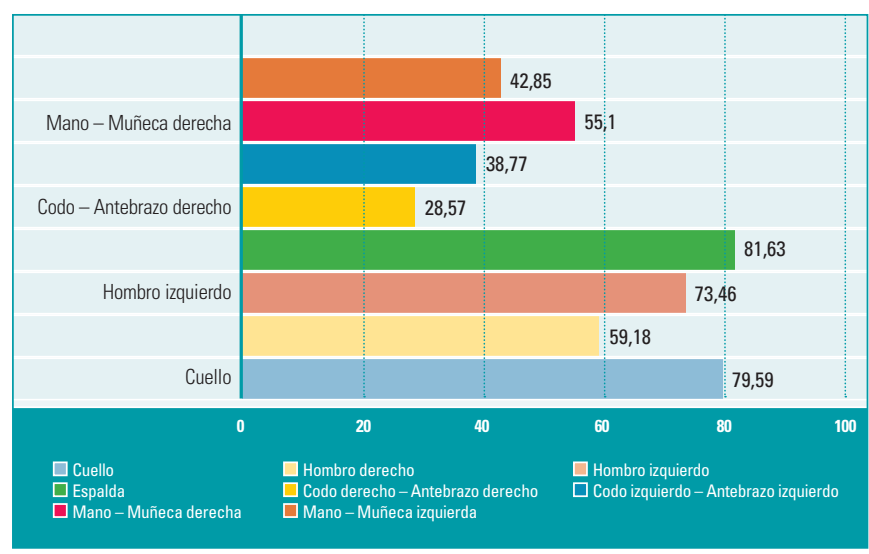

Gráfico 1. Molestias en los últimos 3 meses.

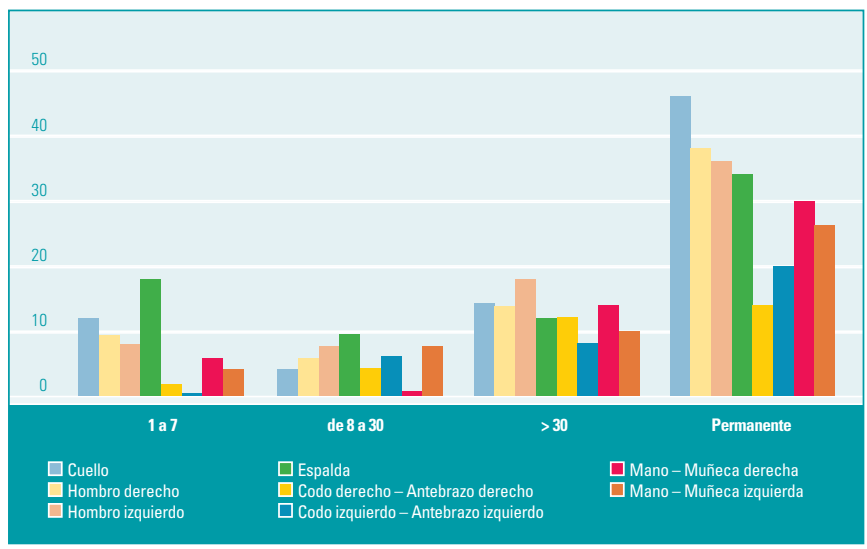

Gráfico 2. Duración de las molestias. 


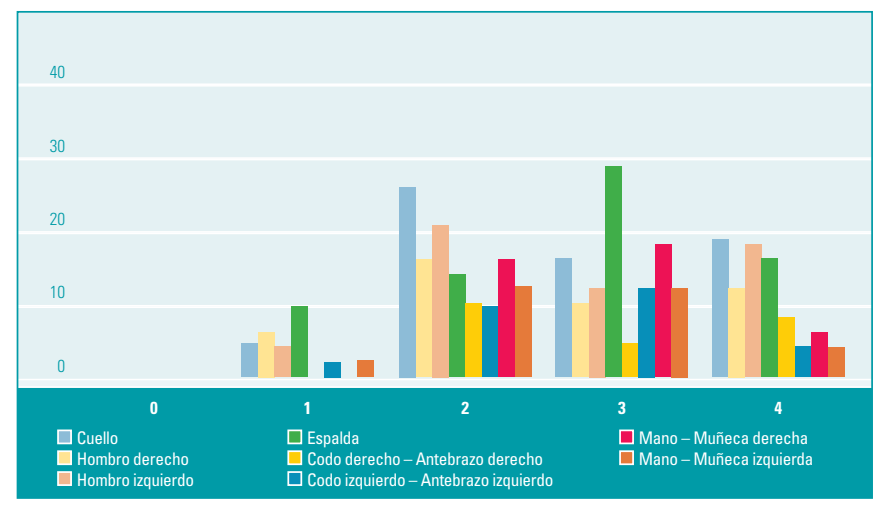

Gráfico 3. Intensidad de las molestias.

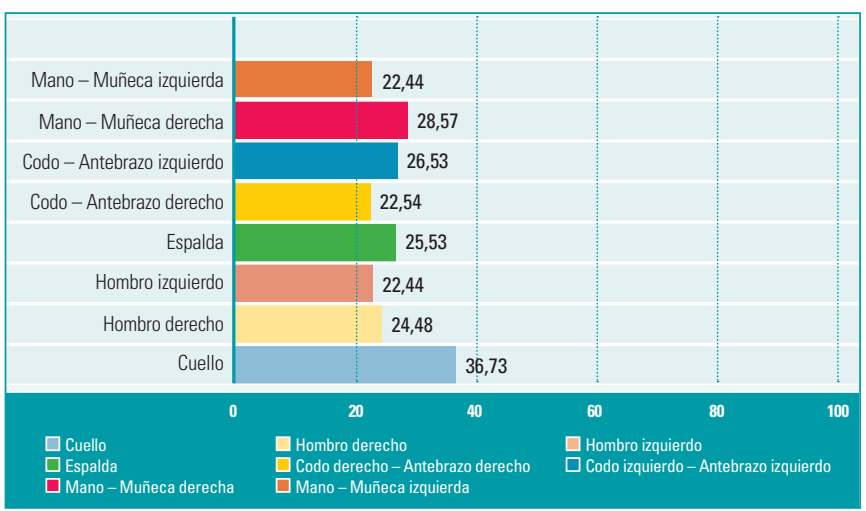

Gráfico 4. Tratamiento médico de los últimos 3 meses.

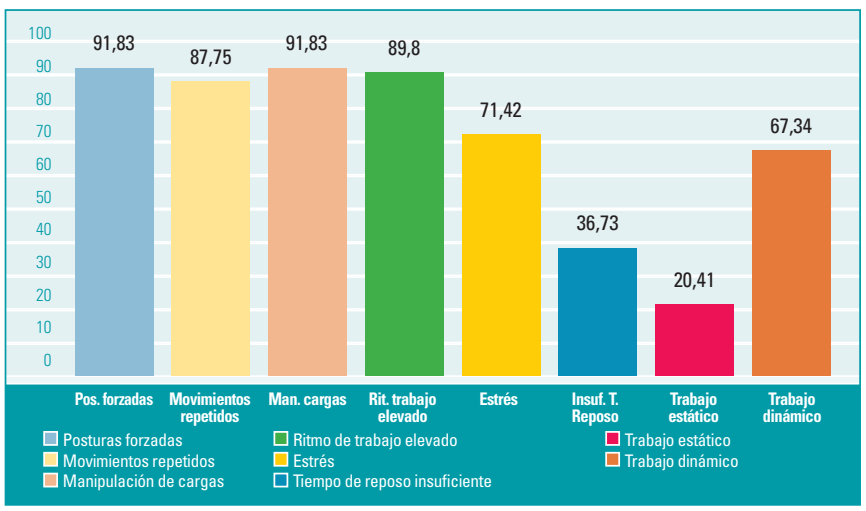

Gráfico 5. Factores en el trabajo.

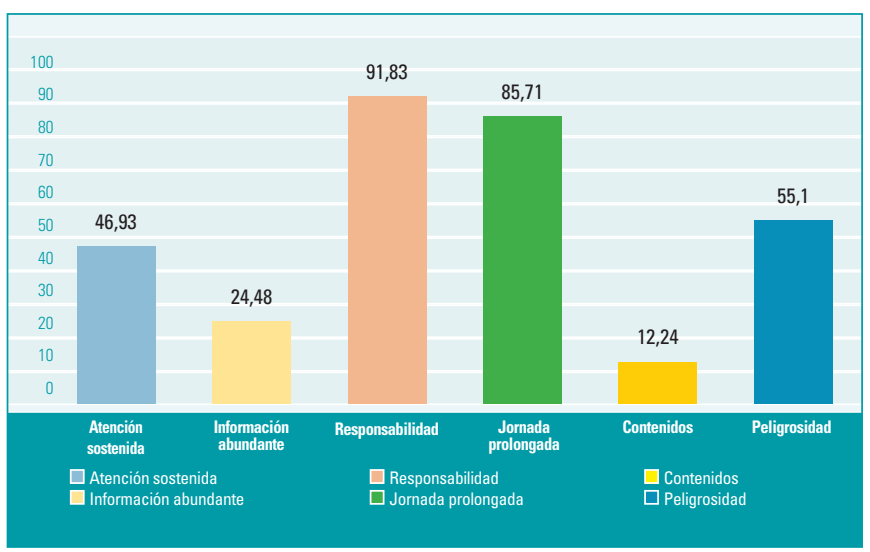

Gráfico 6. Factores procedentes de la exigencia de la tarea.
Una media del $11 \%$ de los encuestados manifiesta tener dolores $y / 0$ molestias de la máxima intensidad (4) en alguna zona corporal.

El ítem original era de respuesta dicotómica (sí/no).

La respuesta no obtuvo un $0 \%$ de resultados. En el gráfico 4 se muestran los resultados, en porcentaje, a la respuesta si en relación con la prescripción de tratamiento médico para un TME en los últimos 3 meses.

Resulta significativo que de estos datos porcentuales se deduce que un 26,27\% de la muestra encuestada ha recibido tratamiento médico para un TME durante el período reseńado.

\section{- FACTORES ORGANIZACIONALES Y PSICOSOCIALES RELACIONADOS CON TRASTORNOS MUSCULOESQUELÉTICOS EN EL CPR MIXTA. GRÁFICOS REPRESENTATIVOS}

El gráfico 5 indica los factores del trabajo relacionados con los TME que los encuestados consideran que están presentes en su centro de trabajo; en este caso, el CPR Mixta.

Las posturas forzadas y la manipulación de cargas son los factores en el trabajo más frecuentes, con un $91,83 \%$ de respuestas, respectivamente; le siguen a continuación el ritmo de trabajo elevado con un 89,8\% y los movimientos repetidos con un $87,75 \%$.

El gráfico 6 indica los factores procedentes de la exigencia de la tarea que los encuestados consideran que están presentes en su desempeńo profesional en el centro de trabajo, en este caso, el CPR Mixta.

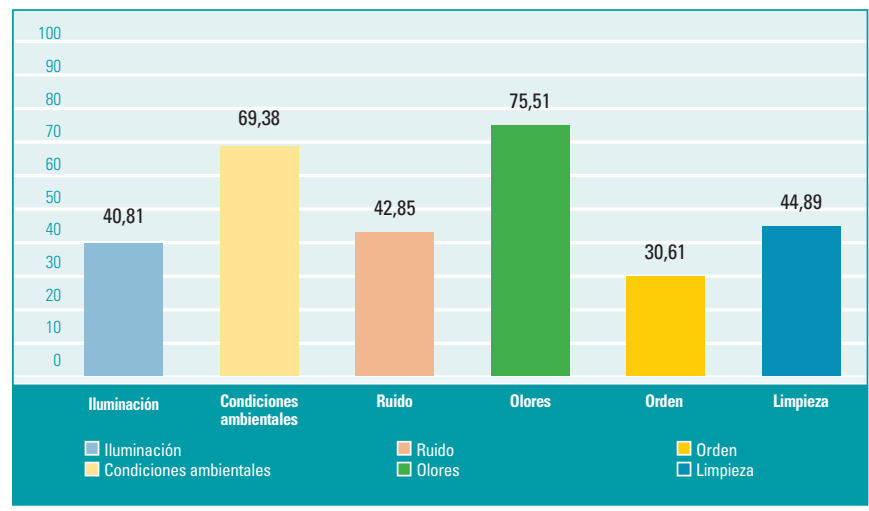

Gráfico 7. Factores procedentes de las condiciones físicas del entorno.

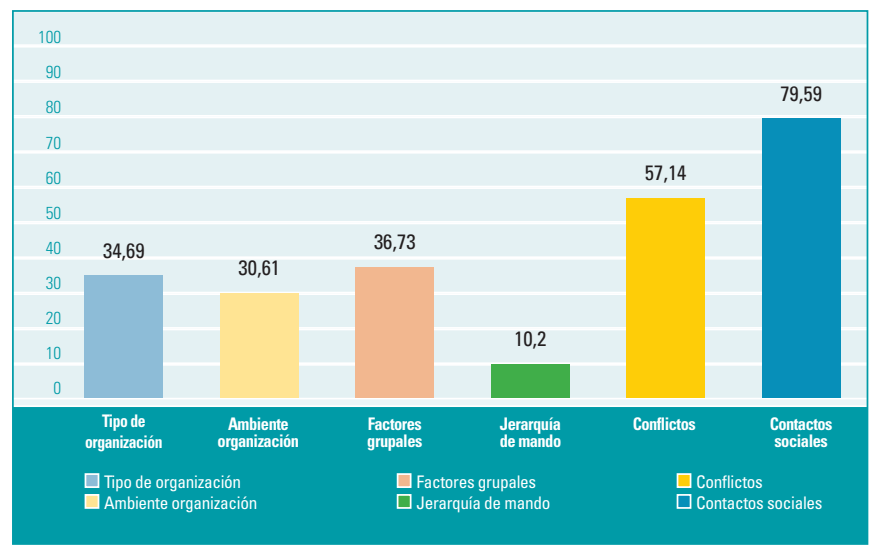

Gráfico 8. Factores sociales y organizativos. 
La responsabilidad, con un $91,83 \%$ de las respuestas, y la jornada prolongada, con un $85,71 \%$, son los factores procedentes de la exigencia de la tarea más destacados; le siguen la peligrosidad con un $55,1 \%$ y la necesidad de atención sostenida con un 46,93\%.

El gráfico 7 indica los factores procedentes de las condiciones físicas del entorno que a los encuestados les parecen inadecuados en su puesto de trabajo, en este caso, el CPR Mixta.

Los olores, con un $75,51 \%$, y las condiciones ambientales (calor, humedad, calidad del aire, etc.), con un 69,38\%, son los aspectos que los encuestados consideran más inadecuados de su puesto de trabajo, seguido de la limpieza con un $44,89 \%$ y el ruido con un $42,85 \%$.

El gráfico 8 indica los factores sociales y organizativos que a los encuestados les generan más carga mental en la empresa; en este caso, el CPR Mixta.

Resulta muy significativo que el factor social y organizativo que genera más carga mental en la empresa según los encuestados sean los contactos sociales (relación con usuarios y clientes), con un 79,59\%, seguido de los conflictos (en el grupo, entre grupos y entre personas), con un 57,14\%, y los factores grupales (estructura y cohesión de grupo), con un 36,72\%.

\section{- DISCUSIÓN}

Los TME en Espańa se sitúan entre las tres primeras causas de baja laboral en aumento continuo. Logran actuar como disparador de una fuerte afectación psicopatológica, y cursan, en la mayoría de los casos, con dolor (6). Cuando hablamos de TME nos referimos a un conjunto de alteraciones sobre cuya denominación ni siquiera los científicos se ponen de acuerdo. Abarcan un amplio abanico de signos y síntomas que pueden afectar a distintas partes del cuerpo: manos, muñecas, codos, nuca, espalda, así como a distintas estructuras anatómicas: huesos, músculos, tendones, nervios, articulaciones. Estas alteraciones no siempre pueden identificarse clínicamente dado que el síntoma clave, el dolor, es una sensación subjetiva y representa muchas veces la única manifestación. Tampoco es extraño que no se puedan catalogar con un diagnóstico preciso: cervicalgia (dolor cervical) o lumbalgia (dolor lumbar) sólo indican la localización anatómica de un síntoma. Su origen debido a múltiples causas y su carácter acumulativo a lo largo del tiempo añaden dificultades a una definición precisa (7).

En el sector sanitario y sociosanitario los riesgos ergonómicos aparecen principalmente por: manipulación manual de cargas (donde destaca la movilización de enfermos, ya que es una de las tareas más frecuentes en el ámbito sanitario); higiene postural (una correcta higiene postural es fundamental para evitar lesiones cuando se lleva a cabo cualquier actividad y aún más en el caso de la manipulación de cargas); movimientos forzados (con o sin carga, pueden provocar contracturas musculares y lesiones de articulaciones y ligamentos); sedentarismo (la falta de actividad física y el sedentarismo provocan debilidad muscular y supone un factor de riesgo ańadido); movimientos imprevistos (si el paciente realiza un movimiento brusco no esperado, es necesario que el trabajador o trabajadora lleve a cabo un sobreesfuerzo que, además, suele hacerse rápidamente y con posturas inadecuadas, con lo que aumenta el riesgo de producirse una lesión) (8).

Un estudio transversal en 133 miembros del personal de enfermería de un hospital geriátrico de EE.UU. analizó la asociación entre el desempeño de éstos en tareas de manipulación y el malestar musculoesquelético. El 62\% de los sujetos notificaron una prevalencia de malestar musculoesquelético de intenso a moderado. La mayor parte de los TME relacionados con el trabajo que se encontraron se relacionaban con trastornos a nivel de espalda, aunque también incluyeron trastornos a nivel de cuello, hombro, brazo, muñeca y rodilla (9). Para el personal de enfermería, la manipulación manual de pacientes (mover o reposicionar a un paciente usando la fuerza del propio cuerpo) es la mayor causa de estos deterioros musculoesqueléticos. Después de tal deterioro, muchos de los trabajadores en salud dejan el sector, de forma temporal o permanente (9).

Las enfermeras a menudo realizan actividades físicas de trabajo pesadas, como el levantamiento de cargas, trabajo en posturas incómodas, transferencia de pacientes, operación de equipos peligrosos, etc. La profesión de enfermero es la segunda en el ranking de carga de trabajo físico, después del trabajo industrial (11). Por esto, el dolor de espalda baja es el TME relacionado con el trabajo que ocurre con mayor frecuencia en esta profesión, con una prevalencia a los 12 meses de entre un $30 \%$ y un $70 \%$. Por otro lado, la tasa de incidencia de dolor de espalda baja de los ayudantes de enfermería es más alta que en ocupaciones tradicionales que tienen las cargas físicas más elevadas, como es el caso de los trabajadores de la construcción y los recolectores de basura (12).

Otro estudio examinó el predominio de los TME en el personal de enfermería en Corea. Los TME relacionados con el trabajo fueron definidos usando tres criterios, basados en la frecuencia, la duración y la intensidad del dolor. Los resultados mostraron que la prevalencia a los 12 meses de TME relacionados con el trabajo en al menos un sitio del cuerpo para los criterios mencionados anteriormente eran del $56,8 \%$, y que el hombro era el más susceptible de generar un TME, seguido de la rodilla, espalda baja, mano/muñeca, cuello, tobillo/pies y dedos de la mano. La prevalencia de TME relacionados con el trabajo era mayor en la unidad de cuidados intensivos, seguido de la sala quirúrgica, y en tercer lugar se ubicó la sala de emergencia (13).

\section{- CONCLUSIONES}

Con este breve estudio se ha pretendido realizar un pequeño acercamiento a la realidad de los TME de este colectivo en el CPR Mixta. Esta aproximación permite confirmar la relación del puesto de trabajo con el padecimiento de TME.

Se confirma la existencia de este tipo de trastornos en un importante porcentaje de profesionales auxiliares de enfermería de este centro, su localización en zonas concretas de los miembros superiores y sus consecuencias sobre la salud laboral en general. Asimismo, se identifican ciertos factores de la tarea, organizativos y psicosociales relacionados directamente con los TME y sobre los que, innegablemente, se puede influir, abriendo por tanto múltiples posibilidades de intervención.

No se puede obviar que este breve estudio presenta carencias metodológicas importantes; realmente, sólo ha pretendido servir de acercamiento a una realidad. Sin embargo, teniendo en cuenta los resultados obtenidos se plantea la necesidad de realizar un estudio más en profundidad sobre los TME en este centro, dirigido a colectivos más extensos y con instrumentos estandarizados. Los objetivos de ese estudio han de ir más allá de la mera obtención de datos estadísticos, deben dirigirse al desarrollo de programas preventivos y formativos para los profesionales $\mathrm{y}$, sobre todo, de intervención ante situaciones que pueden ser susceptibles de modificación. La Guía de Detección, Prevención e Intervención en Patologias Musculoesqueléticas pretende ser un primer paso hacia la consecución de esta meta.

\section{- AGRADECIMIENTOS}

Damos las gracias a todos los profesionales del CPR Mixta que de forma voluntaria participaron en este pequeńo estudio, así como a los responsables del centro que facilitaron el acceso al mismo - 


\section{Anexo I. Cuestionario sobre lesiones musculoesqueléticas en las auxiliares de enfermería del CPR Mixta}

Se presenta a continuación un resumen con algunos de los ítems administrados más significativos del cuestionario original utilizado.

En los últimos 3 meses, ¿has tenido molestias en...?

\begin{tabular}{|l|l|l|}
\hline Parte del cuerpo & & \\
\hline Cuello & & \\
\hline Hombro derecho & & \\
\hline Hombro izquierdo & & \\
\hline Espalda & & \\
\hline Codo - Antebrazo derecho & & \\
\hline Codo - Antebrazo izquierdo & & \\
\hline Mano - Muñeca derecha & & \\
\hline Mano - Muñeca izquierda & & \\
\hline
\end{tabular}

Si todas las respuestas a la pregunta anterior han sido NO, terminar la encuesta.

SEÑALA en el siguiente cuadro con una cruz los factores que se presentan en tu trabajo.

\begin{tabular}{|l|l|}
\hline Posturas forzadas & \\
\hline Movimientos repetidos & \\
\hline Manipulación de cargas & \\
\hline Ritmo de trabajo elevado & \\
\hline Estrés & \\
\hline Insuficientes tiempos de reposo & \\
\hline Trabajo estático & \\
\hline Trabajo dinámico & \\
\hline
\end{tabular}

SEÑALA con una cruz en el siguiente cuadro los factores procedentes de las exigencias de la tarea que se presentan en tu puesto de trabajo.

\begin{tabular}{|l|l|}
\hline Exigencias de la tarea \\
\hline Atención sostenida sobre una o más fuentes de información & \\
\hline Información abundante, variada, compleja, que requiere respuesta & \\
\hline Responsabilidad por la salud o la seguridad de otros & \\
\hline Jornada prolongada, turnos, trabajo nocturno & \\
\hline Contenidos múltiples y complejos & \\
\hline Peligrosidad en las tareas & \\
\hline
\end{tabular}

¡SENAALA con una cruz en el siguiente cuadro los factores procedentes de las condiciones físicas del entorno que te parecen inadecuadas en tu puesto de trabajo.

\begin{tabular}{|l|l|}
\hline Exigencias de la tarea & \\
\hline lluminación & \\
\hline Condiciones ambientales (calor, humedad, calidad del aire,...) & \\
\hline Ruido & \\
\hline Olores & \\
\hline Orden & \\
\hline Limpieza & \\
\hline
\end{tabular}

SEÑALA con una cruz en el siguiente cuadro los factores sociales y organizativos que generan más carga mental en tu empresa.

\begin{tabular}{|l|l|}
\hline Factores sociales y organizativos \\
\hline Tipo de organización (estructura de control y comunicación) & \\
\hline Ambiente en la organización (relaciones y aceptación personal...) & \\
\hline Factores grupales (estructura y cohesión del grupo) & \\
\hline Jerarquía de mando & \\
\hline Conflictos (en el grupo, entre grupos, entre personas) & \\
\hline Contactos sociales (relación con usuarios y clientes) & \\
\hline
\end{tabular}

\section{- BIBLIOGRAFÍA}

1. Fundación Europea para la Mejora de las Condiciones de Vida y de Trabajo, Cuarta encuesta europea sobre las condiciones de trabajo, 2007. Disponible en: http://www. eurofound.europa. eu/ewco/surveys/ EWCS2005/index.htm

2. Martínez Plaza CA. Estrés laboral y trastornos musculoesqueléticos (I). Gestión Práctica de Riesgos Laborales 2009; 61: 38

3. VI Encuesta Nacional de Condiciones de Trabajo. Instituto Nacional de Seguridad e Higiene en el Trabajo (INSHT), 2007. Disponible en: http:// www.insht.es/Observatorio/Contenidos/InformesPropios/Desarrollados/Ficheros/Informe_VI_ENCT.pdf

4. Kuorinka I, Jonsson B, Kilbom A, Vinterberg H, Biering-Sørensen F, Andersson $\mathrm{G}$ y cols. Standardised Nordic questionnaires for the analysis of musculoskeletal symptoms. Applied Ergonomics 1987; 18: 233-37.

5. UGT-Canarias. Secretaría de Salud Laboral y Medio Ambiente. Disponible en: http://www.bvsde.paho.org/bvsacd/cd49/lesiones.pdf
6. Araña Suárez M, Patten SB. Trastornos musculoesqueléticos, psicopatología y dolor. 2011. Disponible en: http:// www.seg-social.es/prdio0/groups/public/documents/binario/143942.pdf

7. Prevención de Riesgos Ergonómicos. Fundación para la prevención de riesgos laborales CCOO. Disponible en: http://www. lexnews.es/wp-content/uploads/2013/05/Manual-para-la-prevencio\%CC \%81n-de-riesgos-ergono \%CC \%81micos-y-psicosociales-en-los-centros-de-atencio\%CC\%81n-a-personas-en-situacio\%CC\%81n-de-dependencia.pdf

8. Guía Básica de Riesgos Laborales específicos en el sector sanitario. Secretaría de salud laboral CCOO. Castilla y León. Disponible en: http://www.castillayleon.ccoo.es/comunes/recursos/6/ pub53319_GUIA_BASICA_DE_RIESGOS_LABORALES_ESPECIFICOS EN EL SECTOR SANITARIO.pdf
9. Daraiseh N, Genaidy A, Karwowski W, Stambough J, Davis LS Huston RK. Musculoskeletal outcomes in multiple body regions and work effects among nurses: the effects of stressful and stimulating working conditions. Ergonomics 2003; 46: 1178-99.

10. Menzel NN, Brooks SM, Bernard TE, Nelson A. The physical workload of nursing personnel: association with musculoskeletal discomfort. Int J Nurs Stud 2004; 41: 859-67.

11. Engels JA, Landeweerd JA, Kant Y. An OWAS-based analysis of nurses' working postures. Ergonomics 1994; 37: 909-19.

12. Marras WS, Davis KG, Kirking BC, Bertsche PK. A comprehensive analysis of low-back disorder risk and spinal loading during the transferring and repositioning of patients using different techniques. Ergonomics 1999; 42: 904-26.

13. Kee D, Seo SR. Musculoskeletal disorders among nursing personnel in Korea. Int J Industrial Ergonomics 2007: 37: 207-12. 